\title{
Utilizing the Shift-or-Shrink Algorithm to Optimize the Direct Coupling from Laser Diodes to Diffused Channel Waveguides
}

\author{
Cheng-Hsiao Chung (D), Chien-Hui Chien (1), Yi-Xuan Wu (D), Chun-Ming Hung (D), \\ Keh-Yi Lee (D)

\begin{abstract}
Electrical Engineering Department, Chinese Culture University, Taiwan, China, konan6918@gmail.com,kitty902220@yahoo.com.tw,a22522683@gmail.com, adam0901200082@,gmail.com,kyleentu@ms52.hinet.net
\end{abstract}

\begin{abstract}
Several formulae for computing the direct coupling efficiencies from laser diodes to diffused channel waveguides, based on mathematic models for approximating the corresponding laser beams and the waveguiding modes, have been derived in this paper. Then, the authors utilize a simple shift-or-shrink (SOS) algorithm to seek their optimal coupling conditions, including the incident positions of the laser beams and the corresponding horizontal and vertical beam widths. The simulation results are presented and compared.
\end{abstract}

Index Terms - Direct coupling efficiency, laser diodes, diffused channel waveguides, algorithm.

\section{INTRODUCTION}

The topics of direct coupling from a laser diode (LD) or an optical fiber to a dielectric channel waveguide are very important in the integrated optics [1]-[14]. Several mathematical models for approximating laser beams radiated from laser diodes and the guided modes of the diffused channel waveguides were also proposed [15]-[18]. For reducing undesired losses due to modal conversion and misalignment between LD and the waveguide, the incident LD's optical beam should have the properest modal profile for fitting the waveguiding mode, and its beam axis has to be aligned to the most appropriate position of the input facet of the channel waveguide. In this way, the optimal coupling efficiency can be achieved. However, the optimization of the direct coupling from LD to the diffused channel waveguide is seldom investigated based on the above mathematical models. In this paper, the authors utilize these models to derive several formulae of the direct coupling efficiencies from LD's optical beams to the diffused channel waveguides. Afterwards, we search for their optimal coupling conditions by a heuristic shift-or-shrink (SOS) algorithm.

SOS algorithm has been proposed for seeking the extrema of a function comprising a few variables, finding the complex solutions of an equation, obtaining the stationary solution in the calculus of variation, and optimizing the photonic device's structures [9], [19], [20]. Among them, the authors have presented an example of searching for the extreme value of a multi-variable paraboloid function by SOS algorithm in [20]. It has been observed that only a few rounds of the shift-or-shrink 
procedures are necessary for finding out the extremum via step-by-step investigation. In this paper, we also utilize SOS algorithm to obtain the optimal incident conditions from LD's optical beam to the diffused channel waveguide. Comparing the other common optimization method such as the genetic algorithm (GA) [21] or particle swarm optimization (PSO) [22], SOS algorithm is capable of providing a sufficiently good solution, and it is more easily developed in computer programming than GA and PSO [20]. This is the reason that the authors select SOS method to seek the optimal incident conditions and its corresponding direct coupling efficiency. In the following paragraph, we also analyze the characteristics of direct coupling efficiency theoretically.

\section{OPTIMIZING THE DIRECT COUPLING EFFICIENCIES OF LASER DIODES TO DIFFUSED CHANNEL WAVEGUIDES BY THE SHIFT-OR-SHRINK ALGORITHM}

Consider the end-butt coupling from LD to a diffused channel waveguide and the corresponding rectangular coordinate system as shown in Fig. 1(a). The horizontal axis is the $x$-axis and the vertically-downward axis is the $y$-axis. The origin of the coordinate system is located at the top surface of the waveguide. The both cross sections of LD and the diffused channel waveguide are symmetric with the $y$-axis. And the notation $c$ denotes the vertical distance from the center of LD's active region to the top surface of the diffused channel waveguide. On the other hand, for improving the direct coupling efficiency, there may exist a tiny lens or lens-like medium for converging the laser beam radiated from LD. Its schematic diagram is shown in Fig. 1(b). The notations $w_{x}$ and $w_{y}$ are respectively the the horizontal and the vertical $e^{-1}$-half-widths of the incident laser beam on the input facet of the diffused waveguide. And $c$ becomes the vertical distance between the incident position of the laser beam and the top surface of the diffused channel waveguide in this sub-figure. The axes of the rectangular coordinate system in Fig. 1(b) is identical to that of Fig. 1(a).

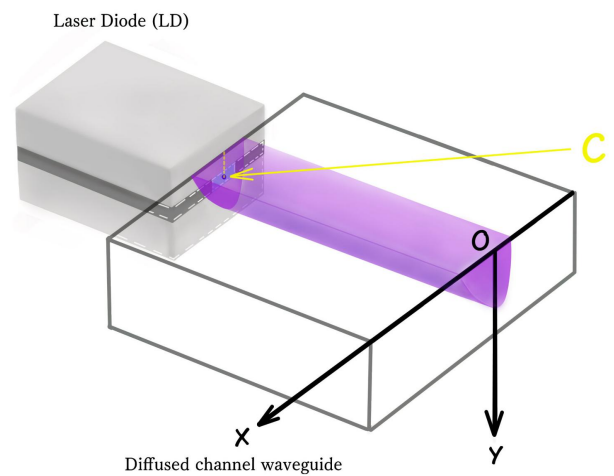

(a)

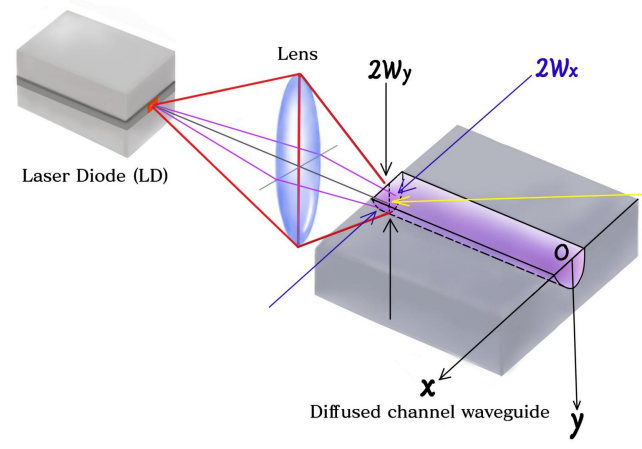

(b)

Fig. 1. (a) The end-butt coupling from a laser diode (LD) to a diffused channel waveguide. (b) The schematic diagram for describing that LD's beam is coupled to a diffused channel waveguide via a tiny converging lens or lens-like medium.

For either cases in sub-figures 1(a) or (b), the direct coupling efficiency between LD's optical beam and the diffused channel waveguide can be calculated by a normalized overlapped integral [8], [9], [14], 


$$
\eta=\frac{\left|\int_{-\infty}^{\infty} \int_{-\infty}^{\infty} \phi(x, y) \varphi^{*}(x, y) d x d y\right|^{2}}{\int_{-\infty}^{\infty} \int_{-\infty}^{\infty}|\phi(x, y)|^{2} d x d y \cdot \int_{-\infty}^{\infty} \int_{-\infty}^{\infty}|\varphi(x, y)|^{2} d x d y}
$$

where $\phi(x, y)$ is the incident optical field radiated by $\operatorname{LD}$ and $\varphi(x, y)$ is the modal function of the diffused waveguide. There are two commonly used mathematical models for approximating optical beams radiated from single-mode laser diodes. One is the elliptically-Gaussian (EG) function. It is widely adopted due to its simplicity. For the coordinate systems as shown in Fig. 1(a) or (b), we can express the normalized modal function of the incident elliptically-Gaussian beam as

$$
\phi_{E G}(x, y)=\sqrt{\frac{2}{w_{x} w_{y} \pi}} \cdot \exp \left(-\frac{x^{2}}{w_{x}^{2}}-\frac{(y-c)^{2}}{w_{y}^{2}}\right)
$$

where $w_{x}$ and $w_{y}$ are the horizontal and the vertical $e^{-1}$-half-widths of the incident laser beam, respectively. And $c$ represents the vertical distance from the incident position of the laser beam to the top surface of the diffused channel waveguide, as an earlier description of Fig. 1(a) and (b). The other model is the Gaussian-Lorentzian (GL) optical beam. It is the product of a Gaussian function in the horizontal direction and a Lorentzian function in the vertical direction. This model is appropriate for approximating an optical beam which is radiated from a single-mode laser diode with an active layer thinner than the operating wavelength [17], [18]. As for this model, we can express its normalized modal function as

$$
\phi_{G L}(x, y)=\sqrt[4]{\frac{8(e-1)}{w_{x}^{2} w_{y}^{2} \pi^{3}}} \cdot \frac{e^{\frac{-x^{2}}{w_{x}^{2}}}}{1+(e-1) \cdot \frac{(y-c)^{2}}{w_{y}{ }^{2}}}
$$

It is easily seen that the optical field expressed as (2b) has the maximum, $\sqrt[4]{\frac{8(e-1)}{w_{x}{ }^{2} w_{y}{ }^{2} \pi^{3}}}$, occurred at $(x=0, y=c)$. Moreover, it becomes $1 / e$ of the maximum in case of $\left(x= \pm w_{x}, y=0\right)$ or $\left(x=0, y=c \pm w_{y}\right)$. That is, $w_{x}$ and $w_{y}$ are its horizontal and the vertical $e^{-1}$-half-widths.

On the other hand, some mathematical models have been proposed for approximating the guided modes of the diffused channel waveguides since their exact analytical solutions are hardly obtained [7], [9], [14]-[16]. One type of approximate model (Type-1 waveguiding mode, W1) has a Gaussian profile in the horizontal direction but two asymmetric half-Gaussian profiles in the vertical direction. Let $a_{1}, a_{2}$, and $a_{3}$ denote the $e^{-1}$-half-widths of the waveguiding mode in the horizontal direction, the vertically-upward direction, and the vertically-downward direction, respectively. For the rectangular coordinate systems as shown in Fig. 1(a) or (b), the normalized waveguiding mode can be expressed as [9], [14], [15] 


$$
\varphi_{W 1}(x, y)=\sqrt{\frac{4}{a_{1}\left(a_{2}+a_{3}\right) \pi}} \cdot e^{\frac{-x^{2}}{a_{1}^{2}}} \cdot e^{\frac{-(y-b)^{2}}{a_{i}^{2}}}, i= \begin{cases}2, & y<b \\ 3, & y \geq b\end{cases}
$$

In this case, the maximum of the waveguiding modal field is located at $x=0$ and $y=b$. Another type of approximate model (Type-2 waveguiding mode, W2) has a horizontal Gaussian profile, as same as Type-1 waveguiding mode. But its vertical optical field is expressed as the first-order HermiteGaussian function in the region $y \geq 0$, but the field does not extend into the region $y<0$ (outside the top surface of the waveguide core). As for this model, the normalized guided modal function can be expressed as [15], [16]

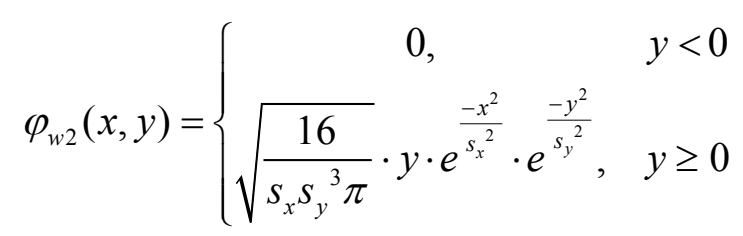

where $s_{x}$ is the horizontal $e^{-1}$-half-width of the waveguiding modal function. And $s_{y}$ is another parameter such that its maximum is located at $x=0$ and $y=s_{y} / \sqrt{2}$.

Substituting (2a) and (3a) into (1), we can obtain the formula of the direct coupling efficiency from LD's elliptically-Gaussian beam to Type-1 waveguiding mode as

$$
\eta_{E G-W 1}=\frac{2 a_{1} w_{x} w_{y}}{\left(a_{2}+a_{3}\right)\left(a_{1}^{2}+w_{x}^{2}\right)} \cdot\left(\frac{a_{2}}{\sqrt{a_{2}^{2}+w_{y}^{2}}} \cdot e^{\frac{-(c-b)^{2}}{a_{2}^{2}+w_{y}^{2}}} \cdot\left\{1-e r f\left[\frac{a_{2}(c-b)}{w_{y} \cdot \sqrt{a_{2}^{2}+w_{y}^{2}}}\right]\right\}+\frac{a_{3}}{\sqrt{a_{3}^{2}+w_{y}^{2}}} \cdot e^{\frac{-(c-b)^{2}}{a_{3}^{2}+w_{y}^{2}}} \cdot\left\{1+e r f\left[\frac{a_{3}(c-b)}{w_{y} \cdot \sqrt{a_{3}^{2}+w_{y}^{2}}}\right]\right\}\right)^{2}
$$

The coupling efficiency expressed in (4a) is a three-variable function of $w_{x}, w_{y}$ and $c$ in case four parameters $a_{1}, a_{2}, a_{3}$ and $b$ are all given. By fundamental principles in Calculus, it is easily shown that the optimal efficiency occurs in the case of $w_{x}=a_{1}$, it means both LD's optical beam and the waveguiding mode have identical horizontal Gaussian profiles. Therefore, we can simplify (4a) into the form

$$
\eta_{E G-W 1, \text { simplified }}=\frac{w_{y}}{a_{2}+a_{3}} \cdot\left(\frac{a_{2}}{\sqrt{a_{2}^{2}+w_{y}^{2}}} \cdot e^{\frac{-(c-b)^{2}}{a^{2}+w_{y}^{2}}} \cdot\left\{1-e r f\left[\frac{a_{2}(c-b)}{w_{y} \cdot \sqrt{a_{2}{ }^{2}+w_{y}{ }^{2}}}\right]\right\}+\frac{a_{3}}{\sqrt{a_{3}^{2}+w_{y}^{2}}} \cdot e^{\frac{-(c-b)^{2}}{a_{3}^{2}+w_{y}^{2}}} \cdot\left\{1+e r f\left[\frac{a_{3}(c-b)}{w_{y} \cdot \sqrt{a_{3}{ }^{2}+w_{y}{ }^{2}}}\right]\right\}\right)^{2}
$$

Expression (4b) becomes a two-variable function of $w_{y}$ and $c$; meanwhile, three parameters $a_{2}, a_{3}$ and $b$ remain but one parameter $a_{1}$ drops. And optimizing (4b) is easier than (4a), so we can save much execution time.

In case we substitute (2a) and (3b) into (1), the formula of the direct coupling efficiency from LD's EG beam to Type-2 waveguiding mode becomes 


$$
\begin{aligned}
& \eta_{E G-W 2}=\frac{32 s_{x} w_{x}}{s_{y}^{3} \pi\left(s_{x}^{2}+w_{x}{ }^{2}\right) w_{y}} \cdot e^{\frac{-2 c^{2}}{s_{y}{ }^{2}+w_{y}{ }^{2}}} \cdot\left\{\frac{s_{y}{ }^{2} w_{y}{ }^{2}}{2\left(s_{y}{ }^{2}+w_{y}{ }^{2}\right)} \cdot e^{\frac{-c^{2} s_{y}{ }^{2}\left(s_{y}{ }^{2}+w_{y}{ }^{2}\right)}{}}\right. \\
& \left.+\frac{\sqrt{\pi} c s_{y}{ }^{3} w_{y}}{2 \cdot \sqrt{\left(s_{y}{ }^{2}+w_{y}{ }^{2}\right)^{3}}} \cdot\left[1+\operatorname{erf}\left(\frac{c s_{y}}{w_{y} \cdot \sqrt{s_{y}{ }^{2}+w_{y}{ }^{2}}}\right)\right]\right\}^{2}
\end{aligned}
$$

It is still a three-variable function of $w_{x}, w_{y}$ and $c$ if the two parameters $s_{x}$ and $s_{y}$ are given. By the same reason, the optimal efficiency occurs in the case of $w_{x}=s_{x}$, and then we have the simplified form

$$
\begin{aligned}
& \eta_{E G-W 2, \text { simplified }}=\frac{16}{s_{y}{ }^{3} \pi w_{y}} \cdot e^{\frac{-2 c^{2}}{s_{y}{ }^{2}+w_{y}{ }^{2}}} \cdot\left\{\frac{s_{y}{ }^{2} w_{y}{ }^{2}}{2\left(s_{y}{ }^{2}+w_{y}{ }^{2}\right)} \cdot e^{\frac{-c^{2} s_{y}{ }^{2}{ }^{2}\left(s_{y}{ }^{2}+w_{y}{ }^{2}\right)}{}}\right. \\
& \left.+\frac{\sqrt{\pi} c s_{y}{ }^{3} w_{y}}{2 \cdot \sqrt{\left(s_{y}{ }^{2}+w_{y}{ }^{2}\right)^{3}}} \cdot\left[1+\operatorname{erf}\left(\frac{c s_{y}}{w_{y} \cdot \sqrt{s_{y}{ }^{2}+w_{y}{ }^{2}}}\right)\right]\right\}^{2}
\end{aligned}
$$

The form expressed in (5b) also becomes a two-variable function of $w_{y}$ and $c$; meantime, one parameter $s_{x}$ drops.

Substituting (2b) and (3a) into (1), we can obtain the direct coupling efficiency of LD's GaussianLorentzian beam and Type-1 waveguiding mode as

$\eta_{G L-W 1}=\frac{8 \sqrt{2(e-1)} \cdot a_{1} w_{x}}{\left(a_{2}+a_{3}\right) \cdot w_{y} \cdot\left(a_{1}{ }^{2}+w_{x}{ }^{2}\right) \cdot \sqrt{\pi^{3}}}\left[\int_{-\infty}^{b} \frac{e^{-\frac{(y-b)^{2}}{a_{2}{ }^{2}}}}{1+(e-1) \cdot \frac{(y-c)^{2}}{w_{y}{ }^{2}}} d y+\int_{b}^{\infty} \frac{e^{-\frac{(y-b)^{2}}{a_{3}{ }^{2}}}}{1+(e-1) \cdot \frac{(y-c)^{2}}{w_{y}{ }^{2}}} d y\right]^{2}$

In this case, we can calculate two integrals in (6a) by some numerical methods. It is also capable of showing that the optimal efficiency occurs in the case of $w_{x}=a_{1}$, and then (6a) can be simplified into

$$
\eta_{G L-W 1, \text { simplified }}=\frac{4 \sqrt{2(e-1)}}{\left(a_{2}+a_{3}\right) \cdot w_{y} \cdot \sqrt{\pi^{3}}} \cdot\left[\int_{-\infty}^{b} \frac{e^{-\frac{(y-b)^{2}}{a_{2}{ }^{2}}}}{1+(e-1) \cdot \frac{(y-c)^{2}}{w_{y}{ }^{2}}} d y+\int_{b}^{\infty} \frac{e^{-\frac{(y-b)^{2}}{a_{3}{ }^{2}}}}{1+(e-1) \cdot \frac{(y-c)^{2}}{w_{y}{ }^{2}}} d y\right]^{2}
$$

Expression (6b) becomes a two-variable function of $w_{y}$ and $c$ with three parameters $a_{2}, a_{3}$ and $b$.

In case we substitute (2b) and (3b) into (1), the direct coupling efficiency from LD's GL beam to Type-2 waveguiding mode becomes

$$
\eta_{G L-W 2}=\sqrt{\frac{2(e-1)}{\pi^{3}}} \cdot \frac{w_{x}}{w_{y} s_{y}{ }^{3}} \cdot \frac{32 s_{x}}{w_{x}{ }^{2}+s_{x}{ }^{2}} \cdot\left[\int_{0}^{\infty} \frac{y e^{-\frac{y^{2}}{s_{y}}}}{1+(e-1) \cdot \frac{(y-c)^{2}}{w_{y}{ }^{2}}} d y\right]^{2}
$$

It is a three-variable function of $w_{x}, w_{y}$ and $c$ with two parameters $s_{x}$ and $s_{y}$. We also can compute the 
integral in (7a) numerically. The optimal efficiency also occurs in the case of $w_{x}=S_{x}$, and then we have

$$
\eta_{G L-W 2 \text {,simplified }}=\sqrt{\frac{2(e-1)}{\pi^{3}}} \cdot \frac{16}{w_{y} s_{y}{ }^{3}} \cdot\left[\int_{0}^{\infty} \frac{y e^{-\frac{y^{2}}{s_{y}{ }^{2}}}}{1+(e-1) \cdot \frac{(y-c)^{2}}{w_{y}{ }^{2}}} d y\right]^{2}
$$

The form expressed in (7b) is a two-variable function of $w_{y}$ and $c$ with one parameter $s_{y}$.

Next, the authors describe how to utilize the shift-or-shrink (SOS) algorithm to seek the respective optimal conditions and their corresponding values of (4a)-(7b) [9], [19], [20]. Consider a general optimization problem of seeking the extremum of a multi-variable function $f\left(x_{1}, x_{2}, x_{3}, \ldots, x_{\mathrm{n}}\right)$ for an arbitrary integer $n$. If an initial value of $h$ and the center $\left(x_{1}, x_{2}, x_{3}, \ldots, x_{\mathrm{n}}\right)$ are given, we can establish the polyhedron with $2 n$ vertices: $\left(x_{1}+h, x_{2}, x_{3}, \ldots, x_{\mathrm{n}}\right),\left(x_{1}-h, x_{2}, x_{3}, \ldots, x_{\mathrm{n}}\right),\left(x_{1}, x_{2}+h, x_{3}, \ldots, x_{\mathrm{n}}\right),\left(x_{1}, x_{2}-h, x_{3}, \ldots, x_{\mathrm{n}}\right)$, $\left(x_{1}, x_{2}, x_{3}+h, \ldots, x_{\mathrm{n}}\right),\left(x_{1}, x_{2}, x_{3}-h, \ldots, x_{\mathrm{n}}\right), \ldots,\left(x_{1}, x_{2}, x_{3}, \ldots, x_{\mathrm{n}}+h\right)$, and $\left(x_{1}, x_{2}, x_{3}, \ldots, x_{\mathrm{n}}-h\right)$. If the extremum of $f\left(x_{1}, x_{2}, x_{3}, \ldots, x_{\mathrm{n}}\right)$ occupies one of these vertices, the polyhedron is shifted such that the new center occupies this vertex. In case the extremum lies on the center of the polyhedron, $h$ is shrunk by a factor such that all the vertices uniformly move toward this center. Once the new polyhedron is generated, we execute the next round of SOS procedure. The whole shift-or-shrink procedures stop when the value of $h$ becomes smaller than a tiny threshold value; meanwhile, the extremum of $f\left(x_{1}, x_{2}, x_{3}, \ldots, x_{\mathrm{n}}\right)$ has been obtained. In this work, we can establish a octahedron in the 3-dimensional space for maximizing the direct coupling function $\eta\left(w_{x}, w_{y}, c\right)$ with three variables, as expressed in (4a), (5a), (6a) or $(7 \mathrm{a})$. The octahedron comprises a center $\left(w_{x}, w_{y}, c\right)$ and six vertices: $\left(w_{x}+h, w_{y}, c\right),\left(w_{x}-h, w_{y}, c\right)$, $\left(w_{x}, w_{y}+h, c\right),\left(w_{x}, w_{y}-h, c\right),\left(w_{x}, w_{y}, c+h\right)$, and $\left(w_{x}, w_{y}, c-h\right)$ in case an intial value of $h$ is given. As for seeking the maximum of the direct coupling function $\eta\left(w_{y}, c\right)$ with two variables, as expressed in (4b), (5b), (6b) or (7b), we just need to reduce the octahedron into the square in the 2-dimensional space, which contains a center $\left(w_{y}, c\right)$ and four vertices: $\left(w_{y}+h, c\right),\left(w_{y}-h, c\right),\left(w_{y}, c+h\right)$ and $\left(w_{y}, c-h\right)$. Among them, all the shift-or-shrink procedures are the same.

\section{NUMERICAL RESULTS}

In this paper, our developed $\mathrm{C} / \mathrm{C}++$ and Matlab programs for simulation are executed in acer's Aspire F15 laptop personal computer. Utilizing SOS algorithm for optimizing (4a), (5a), (6a) and (7a), Table I(a) lists the simulated optimal incident conditions and their corresponding maximum direct coupling efficiencies from the laser diodes to the diffused channel waveguiding modes, based on different models. Four groups of $\left(a_{1}, a_{2}, a_{3}, b\right)$ for Type-1 waveguiding modes and one group of $\left(s_{x}, s_{y}\right)$ for Type-2 waveguiding mode are given in this sub-table because they have been adopted in [7], [9], [14], [15]. 
TABLE I. (A) THE SIMULATED OPTIMAL INCIDENT CONDITIONS AND THE CORRESPONDING DIRECT COUPLING EFFICIENCIES FROM LASER DIODES TO THE DIFFERENT DIFFUSED CHANNEL WAVEGUIDING MODES OBTAINED BY (4a), (5a), (6a) AND (7a).

(B) THE SIMULATED OPTIMAL INCIDENT CONDITIONS AND THE CORRESPONDING DIRECT COUPLING EFFICIENCIES FROM LASER DIODES TO THE DIFFERENT DIFFUSED CHANNEL WAVEGUIDING MODES OBTAINED BY (4b), (5b), (6b) AND (7b).

\begin{tabular}{|c|c|c|c|c|c|c|c|}
\hline \multicolumn{8}{|c|}{$\begin{array}{c}\text { Optimal direct coupling efficiencies from LD's EG beams to } \\
\text { Type-1 waveguiding modes obtained by (4a) }\end{array}$} \\
\hline$a_{1}$ & $a_{2}$ & $a_{3}$ & $b$ & $w_{x}$ & $w_{y}$ & $c$ & $\eta$ \\
\hline 5.10 & 2.10 & 4.60 & 3.10 & 5.10 & 3.35 & 4.11 & $98.41 \%$ \\
\hline 5.00 & 2.10 & 5.00 & 3.00 & 5.00 & & & $98.09 \%$ \\
\hline 4.60 & 2.10 & 3.80 & 2.80 & & & & $99.05 \%$ \\
\hline 3.50 & 2.10 & 2.00 & 2.71 & 3.50 & 2.05 & 2.67 & $99.99 \%$ \\
\hline \multicolumn{8}{|c|}{$\begin{array}{c}\text { Optimal direct coupling efficiency from LD's EG beam to } \\
\text { Type-2 waveguiding mode obtained by (5a) }\end{array}$} \\
\hline$S_{x}$ & $s_{y}$ & & & $w_{x}$ & $w_{y}$ & $c$ & $\eta$ \\
\hline 4.414 & 4.217 & & & 4.414 & 2.723 & 3.389 & $98.23 \%$ \\
\hline \multicolumn{8}{|c|}{$\begin{array}{c}\text { Optimal direct coupling efficiencies from LD's GL beams to } \\
\text { Type-1 waveguiding modes obtained by (6a) }\end{array}$} \\
\hline$a_{1}$ & $a_{2}$ & $a_{3}$ & $b$ & $w_{x}$ & $w_{y}$ & $c$ & $\eta$ \\
\hline 5.10 & 2.10 & 4.60 & 3.10 & 5 & 2.89 & 3.96 & $95.53 \%$ \\
\hline 5.00 & 2.10 & 5.00 & 3.00 & 5.00 & 3.07 & 4.00 & $95.22 \%$ \\
\hline 4.60 & 2.10 & 3.80 & 2.80 & 4.60 & 2.54 & 3.37 & $96.17 \%$ \\
\hline 3.50 & 2.10 & 2.00 & 2.71 & 3.50 & 1.76 & 2.66 & $97.17 \%$ \\
\hline \multicolumn{8}{|c|}{$\begin{array}{l}\text { Optimal direct coupling efficiency from LD's GL beam to } \\
\text { Type-2 waveguiding mode obtained by (7a) }\end{array}$} \\
\hline$S_{x}$ & $s_{y}$ & & & $w_{x}$ & $w_{y}$ & $c$ & $\eta$ \\
\hline 4.414 & 4.217 & & & 4.414 & 2.371 & 3.300 & $94.88 \%$ \\
\hline
\end{tabular}

(a)

\begin{tabular}{cccccc}
\hline \multicolumn{6}{c}{$\begin{array}{l}\text { Optimal direct coupling efficiencies from LD's EG beams to } \\
\text { Type-1 waveguiding modes obtained by (4b) }\end{array}$} \\
\hline $\boldsymbol{a}_{\mathbf{2}}$ & $\boldsymbol{a}_{\mathbf{3}}$ & $\boldsymbol{b}$ & $\boldsymbol{w}_{\boldsymbol{y}}$ & $\boldsymbol{c}$ & $\boldsymbol{\eta}$ \\
\hline 2.10 & 4.60 & 3.10 & 3.35 & 4.11 & $98.41 \%$ \\
2.10 & 5.00 & 3.00 & 3.55 & 4.17 & $98.09 \%$ \\
2.10 & 3.80 & 2.80 & 2.95 & 3.48 & $99.05 \%$ \\
2.10 & 2.00 & 2.71 & 2.05 & 2.67 & $99.99 \%$ \\
\hline
\end{tabular}

Optimal direct coupling efficiency from LD's EG beam to Type-2 waveguiding mode obtained by (5b)

\begin{tabular}{ccccc}
\hline $\boldsymbol{s}_{\boldsymbol{y}}$ & $s_{y} / \sqrt{2}$ & $\boldsymbol{w}_{\boldsymbol{y}}$ & $\boldsymbol{c}$ & $\boldsymbol{\eta}$ \\
\hline 4.217 & 2.982 & 2.723 & 3.389 & $98.23 \%$ \\
\hline
\end{tabular}

Optimal direct coupling efficiencies from LD's EG beams to Type-1 waveguiding modes obtained by $(6 \mathrm{~b})$

\begin{tabular}{cccccc}
\hline $\boldsymbol{a}_{\mathbf{2}}$ & $\boldsymbol{a}_{\mathbf{3}}$ & $\boldsymbol{b}$ & $\boldsymbol{w}_{\boldsymbol{y}}$ & $\boldsymbol{c}$ & $\boldsymbol{\eta}$ \\
\hline 2.10 & 4.60 & 3.10 & 2.89 & 3.96 & $95.53 \%$ \\
2.10 & 5.00 & 3.00 & 3.07 & 4.00 & $95.22 \%$ \\
2.10 & 3.80 & 2.80 & 2.54 & 3.37 & $96.17 \%$ \\
2.10 & 2.00 & 2.71 & 1.76 & 2.66 & $97.17 \%$ \\
\hline
\end{tabular}

Optimal direct coupling efficiency from LD's GL beam to Type-2 waveguiding mode obtained by $(7 \mathrm{~b})$

\begin{tabular}{ccccc}
\hline \multicolumn{6}{c}{ Type-2 waveguiding mode obtained by (7b) } \\
\hline $\boldsymbol{s}_{\boldsymbol{y}}$ & $s_{y} / \sqrt{2}$ & $\boldsymbol{w}_{\boldsymbol{y}}$ & $\boldsymbol{c}$ & $\boldsymbol{\eta}$ \\
\hline 4.217 & 2.982 & 2.371 & 3.300 & $94.88 \%$ \\
\hline
\end{tabular}

(b)

For LD's elliptically-Gaussian beams, all the corresponding coupling efficiencies are beyond 98\% while the optimal incident conditions of $w_{x}, w_{y}$, and $c$ are fulfilled. As for LD's Gaussian-Lorentzian beams, all the optimal coupling efficiencies are beyond 94\%. Among these numerical results, the maximum optical transfer amounts to $99.99 \%$ in the case of coupling EG laser beam to Type-1 waveguiding mode with parameters: $a_{1}=3.50, a_{2}=2.10, a_{3}=2.00$ and $b=2.71$. The reason will be detailedly given in the next paragraph. In addition, all the simulation results show that the optimal 
incident positions are not located at the peaks of the waveguiding modal fields, that is, $c \neq b$ for Type- 1 waveguiding mode and $c \neq s_{y} / \sqrt{2}$ for Type-2 waveguiding mode. Another noticeable phenomenon is $w_{x}=a_{1}$ for the cases of coupling LD's beams to Type- 1 waveguiding modes, and $w_{x}=s_{x}$ for the cases of coupling LD's beams to Type-2 waveguiding modes, regardless of EG or GL beams. These simulation results are in agreement with the results obtained by Calculus in our previous mention. Besides, observing the upper part of Table I(a), it is easily seen that $w_{y}=\left(a_{2}+a_{3}\right) / 2$ is the optimal vertical $e^{-1}$-half-width of LD's elliptically-Gaussian beam as it was coupled to Type-1 waveguiding mode. On the other hand, Table I(b) shows that the simulation results of the optimal efficiencies and their respective incident conditions obtained by $(4 b),(5 b),(6 b)$ and $(7 b)$ are as same as those calculated by (4a), (5a), (6a) and (7a), except deleting $a_{1}, w_{x}$ and $s_{x}$.

Table II lists the execution time ratios of optimizing the simplified expressions as (4b)-(7b) and their corresponding original forms as (4a)-(7a), under the same initial guesses of $h, w_{y}$, and $c$. We present two average values for Type-1 waveguiding modes in this table because there are four groups of $\left(a_{1}, a_{2}, a_{3}, b\right)$. Among Table II, all the time ratios are less than 0.71 . It means that optimizing the simplified functions of fewer variables can save much execution time, as our earlier mention.

TABLE II. THE EXECUTION TIME RATIOS OF OPTIMIZING TWO-VARIABLE DIRECT COUPLING EFFICIENCIES AND THEIR CORRESPONDING THREE-VARIABLE ONES.

\begin{tabular}{cc}
\hline LD's EG beams coupled to Type-1 waveguiding modes & LD's GL beams coupled to Type-1 waveguiding modes \\
\hline Time ratio of optimizing (4b)/optimizing (4a) & Time ratio of optimizing (6b)/optimizing (6a) \\
0.702 (average) & 0.669 (average) \\
\hline LD's EG beam coupled to Type-2 waveguiding mode & LD's GL beam coupled to Type-2 waveguiding mode \\
\hline Time ratio of optimizing (5b)/optimizing (5a) & Time ratio of optimizing (7b)/optimizing (7a) \\
0.554 & 0.632 \\
\hline
\end{tabular}

Fig. 2(a)-(e) present the simulated transverse contours of the modal profiles of the diffused channel waveguides with different waveguiding modal parameters and their corresponding incident LD's beams with the optimal incident conditions. These modal parameters and the optimal incident conditions are the same as those listed in Table I(a). Among them, Fig. 2(d) is worth noticing. It shows that the contour of Type- 1 waveguiding mode with parameters: $a_{1}=3.50, a_{2}=2.10, a_{3}=2.00$ and $b=2.71$ is nearly elliptic, very similar to that of the corresponding optimal incident LD's ellipticallyGaussian beam with conditions: $w_{x}=3.50, w_{y}=2.05$ and $c=2.67$. It gives the reason to explain our previous observation: the maximum power transfer between them is $99.99 \%$ in Table $\mathrm{I}(\mathrm{a})$, because the two contours are in good match such that the value of the overlapped integral (1) almost approaches to unity. Another observable common phenomenon among these sub-figures is that the vertical optical confinement of LD's GL beam is not as good as that of LD's EG beam, so the corresponding optimal direct coupling efficiency from LD's GL beam to the diffused channel waveguide is always less than that between LD's EG beam and the same waveguide. 

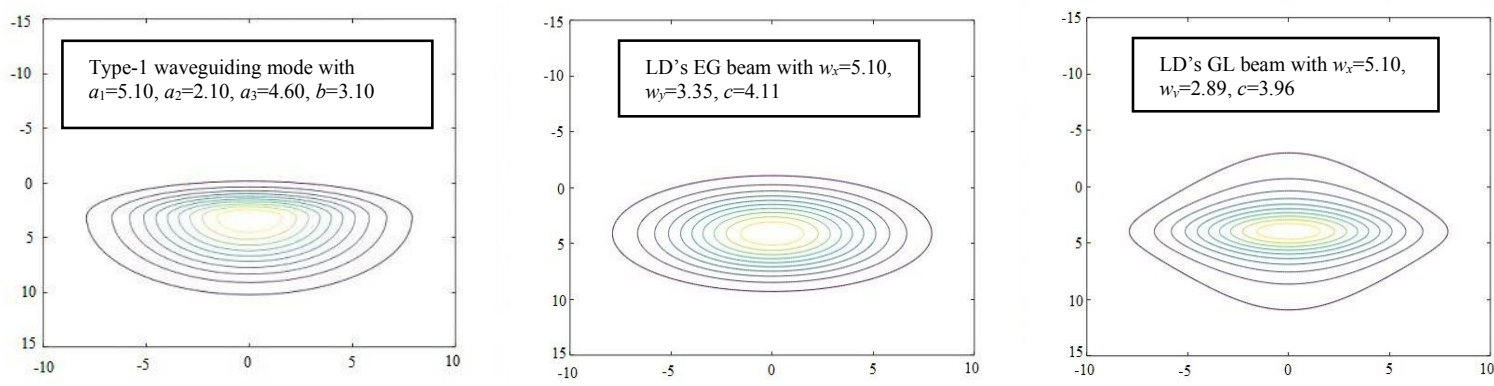

(a)
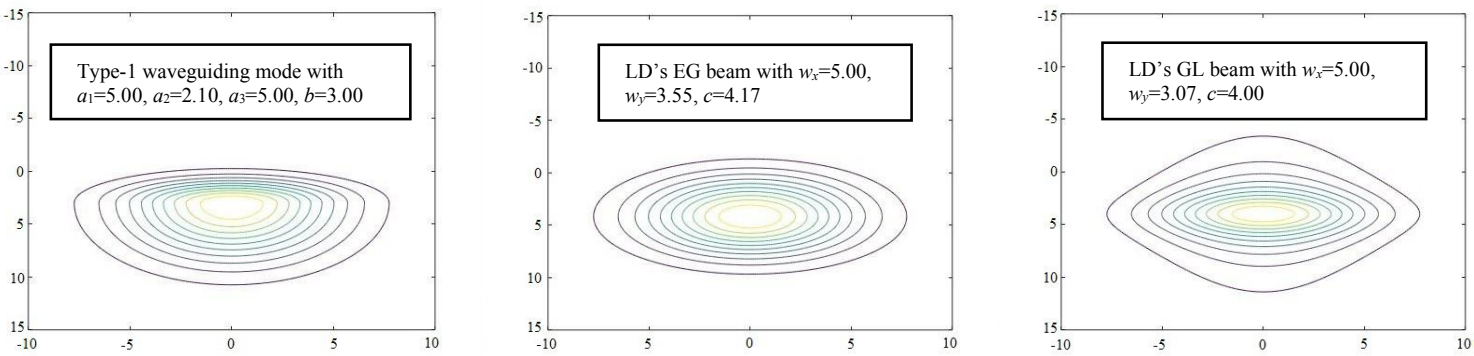

(b)
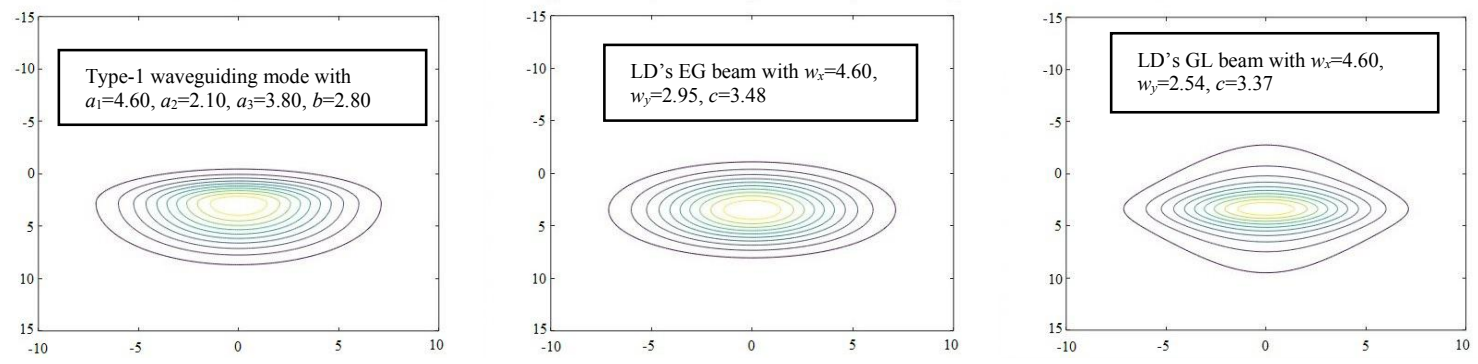

(c)
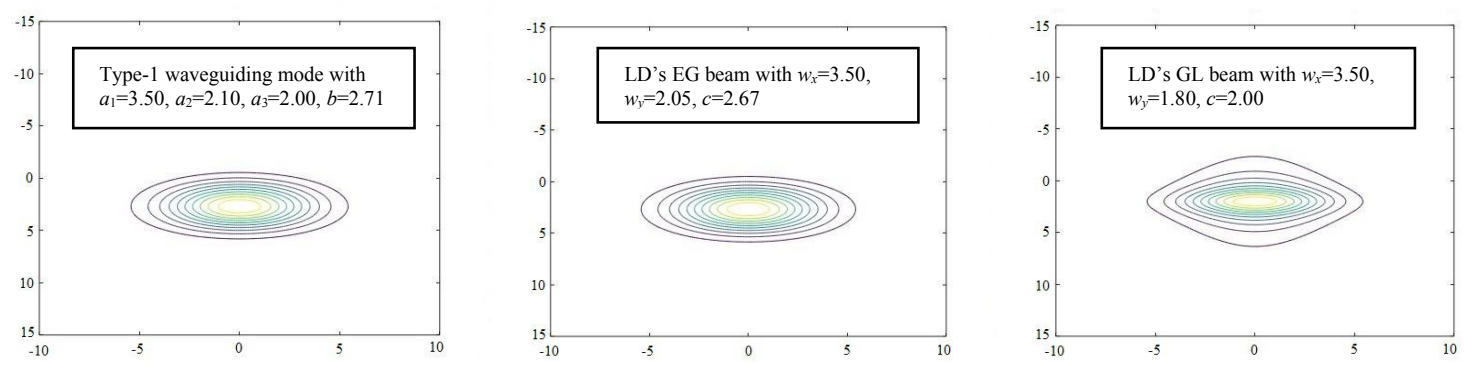

(d)
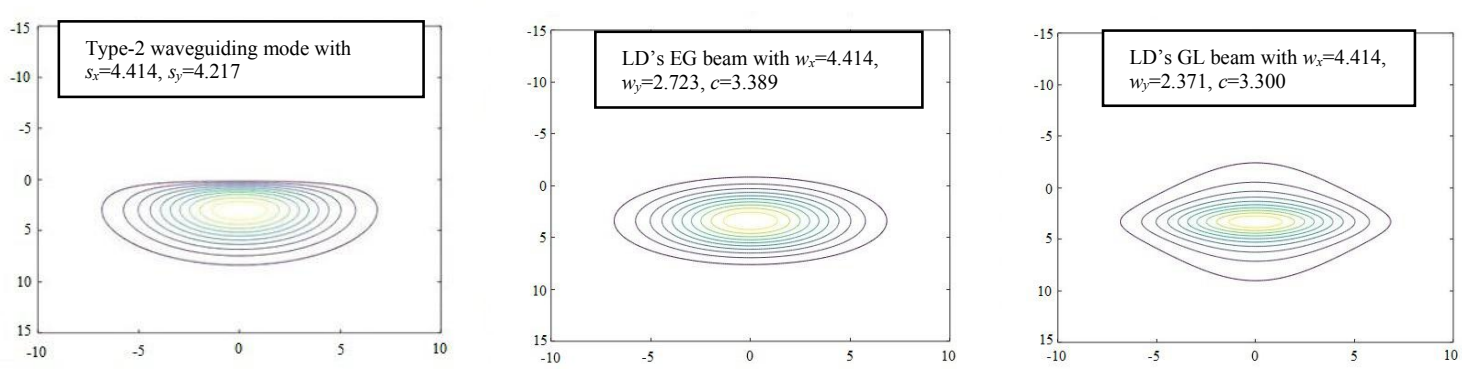

(e)

Fig. 2. The simulated transverse contours of the modal fields of the diffused waveguides and their corresponding optimal incident LD's beams, with the waveguiding modal parameters and the optimized incident conditions listed in Table I(a). 
Consider the case of coupling LD's EG beams to Type-1 waveguiding mode with parameters: $a_{1}=5.10, a_{2}=2.10, a_{3}=4.60$ and $b=3.10$. Fig. 3 (a) describes the simulated variations of the direct coupling efficiency $\eta$ versus the respective deviations of $w_{x}, w_{y}$ and $c$. For the optimal incident conditions: $w_{x}=w_{x 0} \equiv 5.1 \mu m, w_{y}=w_{y 0} \equiv 3.35 \mu m$ and $c=c_{0}=4.11 \mu m$ listed in Table I(a), we set the same deviation range, $\pm 1.5 \mu m$, for $w_{x}, w_{y}$ and $c$ in our simulations. The left sub-figure in Fig. 3(a) shows that $\eta$ increases from $92.73 \%$ to its peak $98.41 \%$ and then decreases to $95.23 \%$, as the value of $\Delta w_{x}$ varies from $-1.5 \mu m$ to $+1.5 \mu m$ around $w_{x 0}$ if we fix $w_{y}=w_{y 0}$ and $c=c_{0}$. The coupling efficiency always keeps beyond $92 \%$ in this interval. In case of the fixed $w_{x}=w_{x 0}$ and $c=c_{0}$, the central sub-figure in Fig. 3(a) describes that $\eta$ grows up from $82.53 \%$ to the maximum $98.41 \%$ and then goes down to $92.12 \%$, as $\Delta w_{y}$ varies within $\pm 1.5 \mu m$ around $w_{y 0}$. It shows that the value of $w_{y}$ smaller than $w_{y 0}$ gives a heavier impact on $\eta$ but the value of $w_{y}$ larger than $w_{y 0}$ affects $\eta$ less. The right sub-figure in Fig. 3(a) presents that $\eta$ is $79.96 \%$ at $\Delta c=-1.5 \mu m$ and becomes $81.13 \%$ at $\Delta c=+1.5 \mu m$ around $c_{0}$, under the fixed $w_{x}=w_{x} 0$ and $w_{y}=w_{y 0}$. It shows that the direct coupling efficiency is more sensitive to the deviation of $c$ than those of $w_{x}$ and $w_{y}$. Therefore, carefully adjusting the incident position of the laser beam by utilizing modern techniques is very important. As for the case of LD's EG beams being coupled to Type-2 waveguiding mode with parameters: $s_{x}=4.414$ and $s_{y}=4.217$, Fig. 3(b) presents the variations of the coupling efficiencies versus the respective deviations of the incident conditions of LD's EG beams around the optimal incident conditions: $w_{x}=w_{x 1} \equiv 4.414, w_{y}=w_{y 1} \equiv 2.723$ and $c=c_{1} \equiv 3.389$. The simulation results show that the dependences of $\eta$ versus $\Delta w_{x}, \Delta w_{y}$ and $\Delta c$ have similar phenomena to those shown in Fig. 3(a). The value of $\eta$ is also the most sensitive to $\Delta c$ among them. Another two cases: the dependences of $\eta$ versus $\Delta w_{x}, \Delta w_{y}$ and $\Delta c$ for the direct coupling of LD's GL beams and Type-1 waveguiding mode with parameters: $a_{1}=5.10, a_{2}=2.10, a_{3}=4.60$ and $b=3.10$ are depicted in Fig. 3(c), and those for the direct coupling from LD's GL beams to Type-2 waveguiding mode with parameters: $s_{x}=4.414$ and $s_{y}=4.217$ are shown in Fig. 3(d). Their behaviors are similar to those shown in Fig. 3(a) and (b). For simplicity, the other cases of coupling LD's beams to Type-1 waveguiding mode with the other parameters are omitted in this work. 

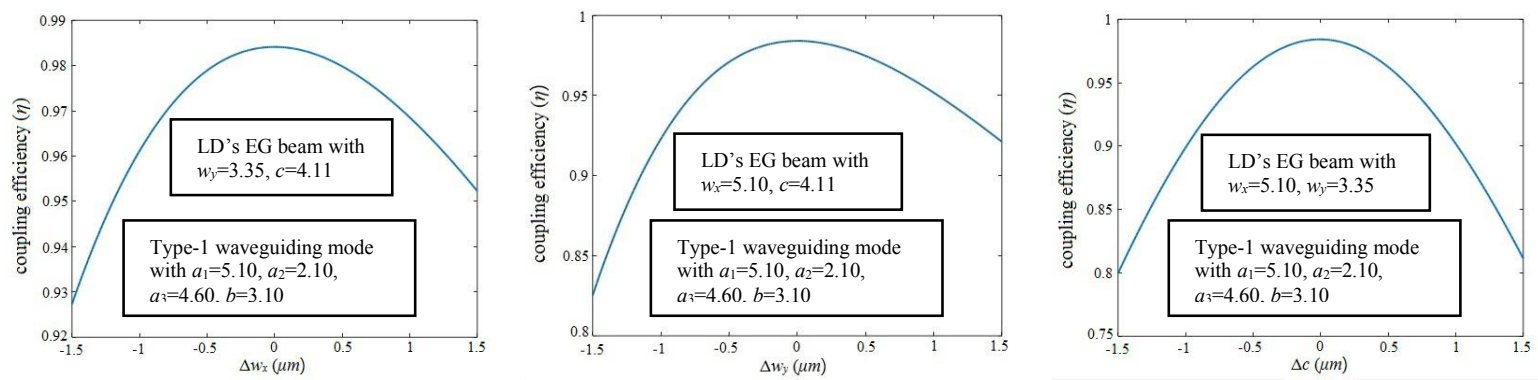

(a)
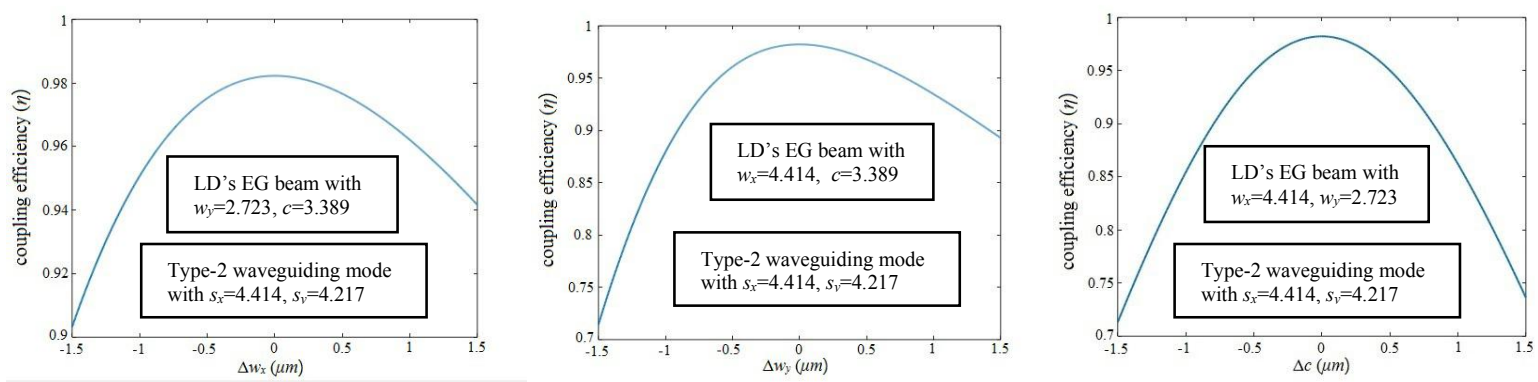

(b)
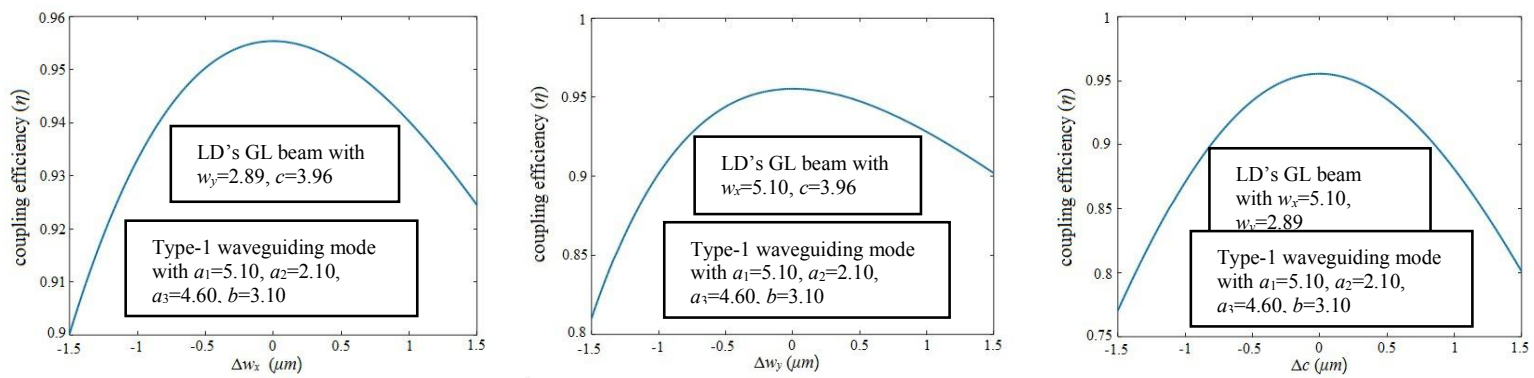

(c)
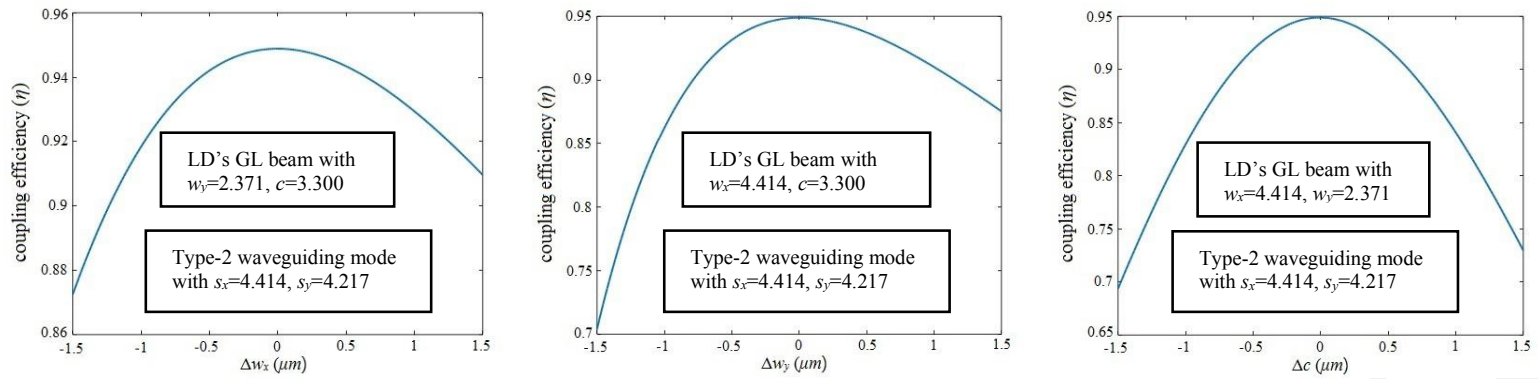

(d)

Fig. 3. The simulated variations of the direct coupling efficiency $(\eta)$ versus the deviations $\Delta w_{x}, \Delta w_{y}$ and $\Delta c$ in case of (a) coupling LD's EG beam to Type-1 waveguiding mode with $a_{1}=5.10, a_{2}=2.10, a_{3}=4.60$ and $b=3.10$. (b) coupling LD's EG beam to Type-2 waveguiding mode with $s_{x}=4.414$ and $s_{y}=4.217$. (c) coupling LD's GL beam to Type-1 waveguiding mode with $a_{1}=5.10, a_{2}=2.10, a_{3}=4.60$ and $b=3.10$. (d) coupling LD's GL beam to Type- 2 waveguiding mode with $s_{x}=4.414$ and $s_{y}=4.217$. 


\section{CONCLUSIONS}

We draw some conclusions from our simulation results as follows. For coupling LD's ellipticallyGaussian beams with parameters $\left(w_{x}, w_{y}, c\right)$ to Type- 1 waveguiding mode with parameters $\left(a_{1}, a_{2}, a_{3}, b\right)$, the optimal conditions always occurs in case of $w_{x}=a_{1}$ and $w_{y}=\left(a_{2}+a_{3}\right) / 2$. However, the optimal value of $c$ cannot be expressed in a simple analytical function of $a_{1}, a_{2}, a_{3}$ and $b$. In this case, all the optimal coupling efficiencies are beyond 98\%. Especially, the maximum power transfer between them amounts to $99.99 \%$ in case the optimal transverse contour of the incident LD's laser beam is similar to that of the waveguiding mode. Besides, the optimal incident position of laser beam's axis is not located at the maximum of the waveguiding modal field, i.e., $c \neq b$. As for coupling LD's EG beams to Type- 2 waveguiding mode with parameters $\left(s_{x}, s_{y}\right)$, we have the optimal condition $w_{x}=s_{x}$, but $w_{y}$ is not capable of being expressed in an analytical form of $s_{x}$ and $s_{y}$. In this case, the optimal incident position of laser beam's axis is still not situated at the maximum of the waveguiding mode, i.e., $c \neq s_{y} / \sqrt{2}$. On the other hand, for coupling LD's Gaussian-Lorentzian beams with parameters $\left(w_{x}, w_{y}, c\right)$ to Type-1 waveguiding mode with parameters $\left(a_{1}, a_{2}, a_{3}, b\right)$, we also have optimal condition $w_{x}=a_{1}$, but the values of the optimal $w_{y}$ and $c$ are not the simple analytical functions of $a_{1}, a_{2}, a_{3}$ and $b$. As for coupling LD's GL beams to Type- 2 waveguiding mode with parameters $\left(s_{x}, s_{y}\right)$, the optimal condition $w_{x}=S_{x}$ is still valid. The corresponding optimal direct coupling efficiency from LD's GL beam to the diffused channel waveguide is always less than that from LD's EG beam to the same waveguide, and it means that the EG beam provides a better match for the diffused channel waveguiding mode than GL beam. Furthermore, the simulation results also show that the direct coupling efficiency is more sensitive to the deviation of $c$ than those of $w_{x}$ and $w_{y}$.

\section{REFERENCES}

[1] R. G. Hunsperger, A. Yariv, and A. Lee, "Parallel end-butt coupling for optical integrated circuits," Appl. Opt., vol. 16, pp. 1026-1032, 1977.

[2] D. G. Hall, R. R. Rice, and J. D. Zino, "Simple Gaussian-beam model for GaAlAs double-heterostructure laser-diodeto-diffused-waveguide coupling calculations," Opt. Lett., vol. 4, pp. 292-294, 1979.

[3] C. Mueller, C. Sullivan, W. Chang, D. Hall, J. Zino, and R. Rice, "An analysis of the coupling of an injection laser diode to a planar $\mathrm{LiNbO}_{3}$ waveguide," IEEE J. Quantum Electron., vol. QE-16, pp. 363-372, 1980.

[4] P. D. Bear, "Microlenses for coupling single-mode fibers to single-mode thin-film waveguides," Appl. Opt., vol. 19, pp. 2906-2909, 1980.

[5] J. M. Hammer, D. Botez, C. C. Neil, and J. C. Connolly, "High-efficiency high-power butt coupling of single-mode diode lasers to indiffused $\mathrm{LiNbO}_{3}$ optical waveguides," Appl. Phys. Lett., vol. 39, pp. 943-945, 1981.

[6] J. M. Hammer and C. C. Neil, "Observations and theory of high-power butt coupling to $\mathrm{LiNbO}_{3}$-type waveguides," IEEE J. Quantum Electron., vol. QE-18, pp. 1751-1758, 1983.

[7] E. J. Murphy, T. Rice, L. McCaughan, G. Harvey, and P. Read, "Permanent attachment of single-mode fiber arrays to waveguides," IEEE J. Lightwave Technol., vol. LT-3, pp. 795-799, 1985.

[8] K.-Y. Lee and W.-S. Wang, "Non-Gaussian beams in direct coupling from laser diodes into optical waveguides via small gaps" Microwave Opt. Technol. Lett., vol. 5, pp. 99-101, 1992.

[9] Keh-Yi Lee, Chyi-Jieh Hsieh, Jyh-Rou Sze, and Gwo-Jiunn Jaw, "Optimal direct coupling from single-mode fibers to Ti:LiNbO 3 channel waveguides,” Fiber Integrated Opt., vol. 15, pp. 365-371, 1996.

[10] Kai-Ning Ku and Ming-Chang M. Lee, "Cascade of two opposite tapers for butt-coupling between fibers and silicon photonic wires with large misalignment tolerance and low polarization dependency," 2013 Optical Fiber Communication Conference and Exposition and the National Fiber Optic Engineers Conference (OFC/NFOEC), 2013.

[11] X.-F. Yang, Z.-B. Zhang, W.-Y. Du, Q. Zhang, W. Wong, D.-Y. Yu, E. Y.-B. Pun, and D.-L. Zhang, "Nearstoichiometric TiScLiNbO 3 strip waveguide for integrated optics," Opt. Materials Express, vol. 6, pp. 2637-2643, 2016.

[12] G. Son, S. Han, J. Park, K. Kwon, and K. Yu "High-efficiency broadband light coupling between optical fibers and photonic integrated circuits" Nanophotonics, vol. 7, pp. 1845-1864, 2018. 
[13] B. N. Carnio and A. Y. Elezzabi, "Off-normal incidence coupling for perfectly phase-matched second harmonic generation in a sub-micron $\mathrm{LiNbO}_{3}$ planar waveguide," IEEE J. Lightwave Technol., vol. LT-38, pp. 3959 - 3964, 2020.

[14] Y.-X. Wu, C.-H. Chien, C.-H. Chung, C.-M. Hung, and K.-Y. Lee, "Optimizing direct coupling between laser diode and channel waveguide by shift-or-shrink (SOS) algorithm," 2020 Optics \& Photonics Taiwan International Conference (OPTIC 2020), 2020.

[15] N. H. Zhu and R. M. Wang, "A simple variational analysis of diffused channel waveguides," Opt. Quantum Electron., vol. 25 pp. 57-62, 1993.

[16] A. Shanna and P. Bindal, "Analysis of diffused planar and channel waveguides, " IEEE J. Quantum Electron., vol. QE29, pp. 150-153, 1993

[17] W. P. Dumke, "The angular beam divergence in double-heterojunction lasers with very thin active regions," IEEE $J$. Quantum Electron., vol. QE-11, pp. 400-402, 1975.

[18] A. Naqwi and F. Durst, "Focusing of diode laser beams: a simple mathematical model," Appl. Opt., vol. 29, pp. 17801785,1990

[19] K.-Y. Lee, J.-R. Sze, and C.-J. Hsieh, "Variational analysis of ridge-type Ti:LiNbO 3 waveguides by a heuristic algorithm," J. Opt. Commun., vol. 18, pp. 104-108, 1997.

[20] R.-C. Lu and K.-Y. Lee, "Utilizing shift-or-shrink (SOS) method to optimize multi-segment structures of bent optical waveguides," J. Micro., Optoelec. and Elec. App., vol. 18, pp. 316-327, 2019.

[21] John H. Holland, "Adaptation in natural and artificial systems: an introductory analysis with applications to biology," Control, and artificial intelligence. MIT press, 1992.

[22] H. Mouna, V. Mekaladevi, M. N. Devi, "Design of microwave absorbers using improvised particle swarm optimization algorithm", J. Micro., Optoelec. and Elec. App., vol. 17, pp. 188-200, 2018. 